\title{
Anatomía Cardíaca de Pontoporia blainvillei
}

\author{
Cardiac Anatomy of the Pontoporia blainvillei
}

William Pérez \& Martin Lima

PÉREZ, W. \& LIMA, M. Anatomía cardíaca de Pontoporia blainvillei. Int. J. Morphol., 24(3):351-356, 2006.

RESUMEN: El objetivo de este estudio fue describir la conformación interna y externa y la irrigación del corazón del delfín franciscana de la Plata (Pontoporia blainvillei) para lo cual fueron disecados 12 corazones. El pericardio se fijaba caudalmente al diafragma, ventralmente al músculo transverso torácico y, lateralmente, a las costillas y músculos intercostales. El corazón era aplastado dorsoventralmente, su base era craneal, su ápex era compartido por ambos ventrículos. El ventrículo izquierdo era de pared gruesa, mientras que la del ventrículo derecho más delgada y depresible. La cara ventral correspondía a la cara auricular y la cara dorsal a la cara atrial de la Nomina Anatomica Veterinaria, 2005 (NAV). En la conformación interior del atrio derecho destacaba el gran desarrollo de la cresta terminal y de la fosa oval. Dentro del ventrículo derecho, los músculos papilares se distribuían en subbarterioso, magnus y parvi. Una trabécula septomarginal se extendía entre los músculos papilares subarterioso y magnus. También fueron observadas trabéculas menores del mismo tipo, situadas más ventralmente a la anterior. Las venas pulmonares se unían en un tronco común antes de llegar al atrio izquierdo. El ventrículo izquierdo tenía dos músculos papilares bien desarrollados. La irrigación cardíaca arterial mostraba un predominio de la arteria coronaria izquierda y se notaba la presencia de anastomosis intercoronarias.

PALABRAS CLAVE: Delfín del Plata; Anatomía cardíaca; cardiología; Arterias coronarias.

\section{INTRODUCCIÓN}

El delfín franciscana, (Pontoporia blainvillei) del Orden Cetacea, se halla a lo largo de las costas de Sudamérica desde el sur de Brasil hasta Península Valdez en Argentina y, debido a que se encuentra mayormente en el estuario del Río de la Plata, su nombre común es "delfín del plata".

Muy pocos artículos de la anatomía del corazón de Cetáceos han sido publicados y menos en delfines. En un trabajo (Kamiya, \& Yamasaki, 1974) se estudió el peso del órgano en relación al peso corporal en Pontoporia blainvillei de Uruguay. No fue posible encontrar otra publicación del corazón de Pontoporia blainvillei. Recientemente, Van Nie (Van Nie, 1985) estudió el sistema de conducción en Lagenorhynchus albirostris.

El objetivo de este trabajo fue hacer una descripción anatómica general del corazón Pontoporia blainvillei y proponer adaptarle la terminología de la Nomina Anatomica Veterinaria, 2005 (NAV).

\section{MATERIAL Y MÉTODO}

Doce delfines franciscana (Pontoporia blainvillei), capturados accidentalmente por las redes de barcos pesqueros en el Río de la Plata, fueron necropsiados y aislados sus órganos para diversos estudios. Los corazones fueron estudiados in situ y después de retirados fueron colocados en solución de formol al $10 \%$. Luego, fueron estudiados por medio de disección simple, observando primero la conformación exterior. Posteriormente, se disecaron las arterias coronarias y su conformación interior. Se fotografiaron los corazones in situ y aislados. Para facilitar la visualización de sus arterias, dos corazones fueron inyectados con látex coloreando las arterias coronarias en los senos de la aorta. Se utilizó la nomenclatura de la Quinta Edición de la NAV.

\section{RESULTADOS}

La cavidad torácica de Pontoporia blainvillei estaba dividida en tres partes mayores, una parte ventral o parte 
cardíaca, donde se localizaba el corazón (Fig. 1) rodeado por una vasta cavidad pericárdica (Fig. 1). Dorsalmente, habían dos partes pulmonares ocupadas por los pulmones rodeados por las cavidades pleurales.

El pericardio estaba ventralmente a las cavidades pleurales, y los pulmones no cubrían lateralmente al pericardio. El pericardio se unía caudalmente al diafragma, ventralmente al músculo transverso torácico y lateralmente a las costillas y músculos intercostales. Cranealmente en la base del corazón la hoja parietal del pericardio seroso se reflejaba para continuarse con el epicardio. La reflexión avanzaba sobre $5,0 \mathrm{~cm}$ en la aorta y el tronco pulmonar, quedando incluida la porción inicial de esos vasos en la cavidad pericárdica. Sobre las aurículas, la línea de reflexión estaba más próxima al corazón y ambas aurículas se veían como colocadas en dos fondos de saco craneales de la cavidad pericárdica.

El color del corazón en los animales más frescos era rojo oscuro. Los surcos coronario e interventriculares estaban casi desprovistos de grasa. La consistencia del corazón era firme. El ventrículo izquierdo (2, Figs. 1, 2 y 3) tenía la pared mucho más gruesa que el derecho que, a su vez era delgado y depresible. El espesor de la pared del ventrículo derecho (1, Figuras 1,2 y 3 ) era de $6,0 \mathrm{~mm}$ y de $12,0 \mathrm{~mm}$ para el izquierdo en su parte más espesa, por la mitad de su largo. Los atrios eran bastante similares en consistencia y espesor. El peso del corazón aislado promedió los 115 g.

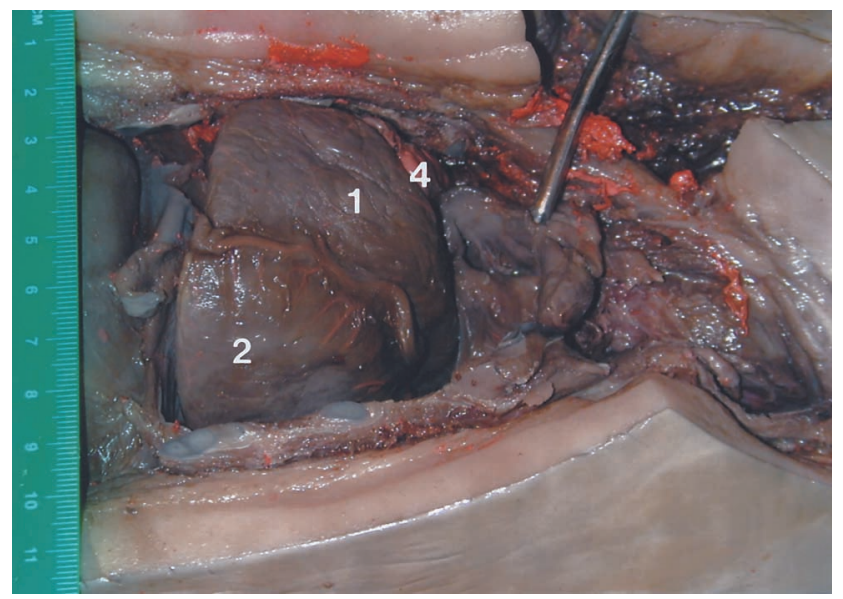

Fig. 1. Vista ventral del corazón in situ tras apertura del tórax de Pontoporia blainvillei.

Referencias numéricas de las 5 Figs. 1. Ventrículo derecho; 2. Ventrículo izquierdo; 3. Aurícula izquierda; 4. Aurícula derecha; 5. Tronco pulmonar; 6. Aorta; 7. Surco y rama interventricular paraconal; 8. Surco y rama interventricular subsinusal; 9. Músculos pectinados; 10. Cresta terminal; 11. Fosa oval; 12. Músculo papilar; 13. Grasa subendocárdica; 14. Orificio atrioventricular derecho; 15. Orificio pulmonar.

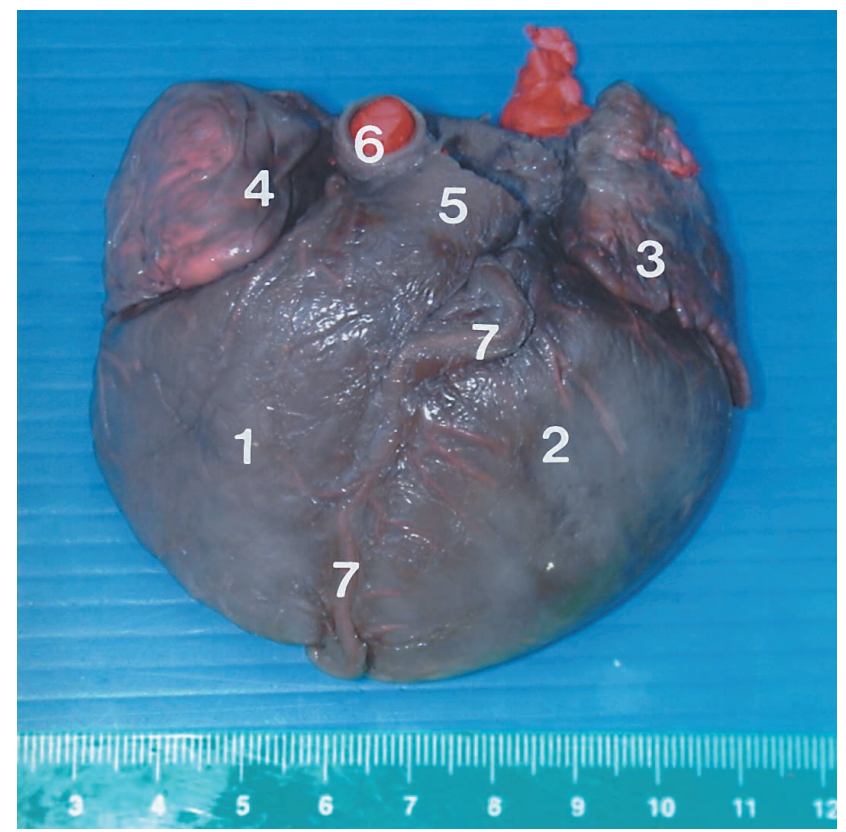

Fig. 2. Cara auricular del corazón de Pontoporia blainvillei.

La forma del corazón era similar a un triángulo equilátero, se le describen una base, dos caras, dos bordes y un ápex.

El corazón estaba aplastado dorsoventralmente, su cara ventral era convexa y su cara dorsal plana (Figs. 1 y 2 ). La base del corazón se situaba cranealmente (Fig. 1) y emi-

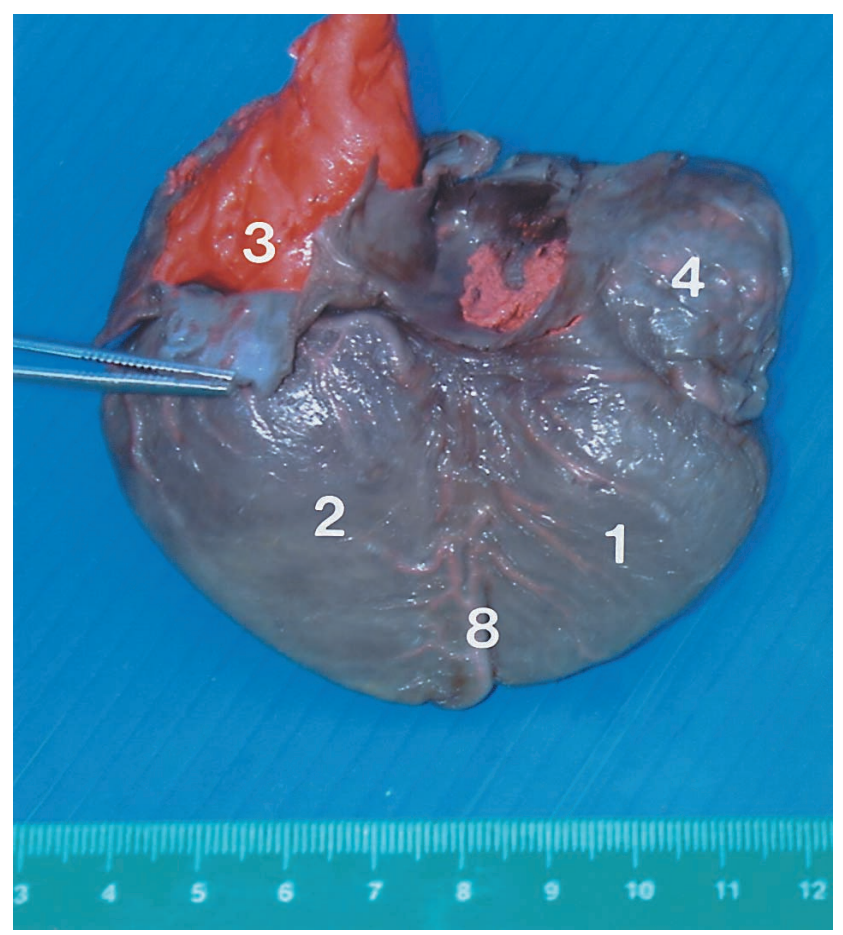

Fig. 3. Cara atrial del corazón de Pontoporia blainvillei. 


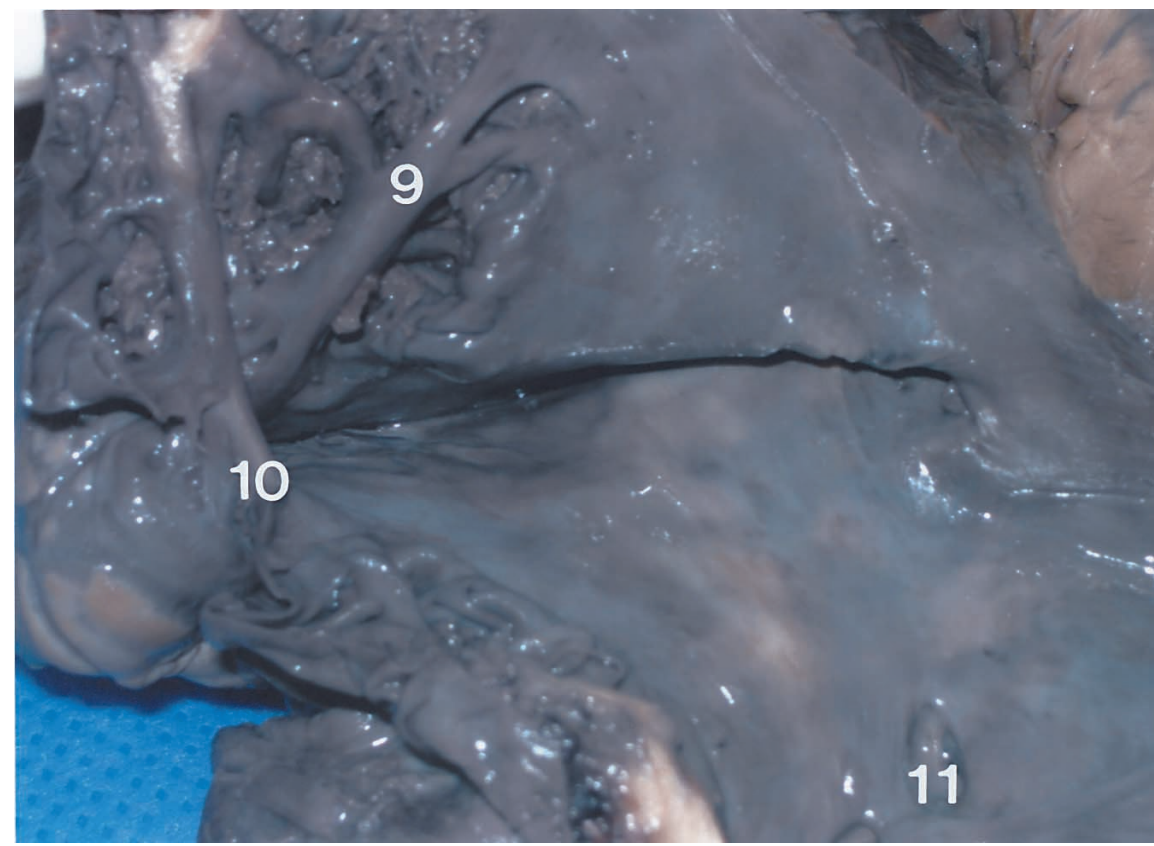

Figure 4. Interior del atrio derecho incidido del corazón de Pontoporia blainvillei.
La cara ventral del corazón (Fig. 2) estaba dividida por el surco interventricular paraconal (7, Fig. 2) que surgía del surco coronario hacia la izquierda y que dividía completamente a la parte ventricular de esta cara en los dos ventrículos, hacia la derecha el ventrículo derecho y hacia la izquierda el ventrículo izquierdo.

El surco coronario estaba interrumpido en el nivel de emergencia del tronco pulmonar (5, Fig. 2). En la cara atrial (Fig. 3) este surco dividía completamente atrios de ventrículos. El surco interventricular subsinusal $(8$, Fig. 3) dividía completamente la parte ventricular de la cara atrial en los dos ventrículos situados hacia los costados respectivos del animal.

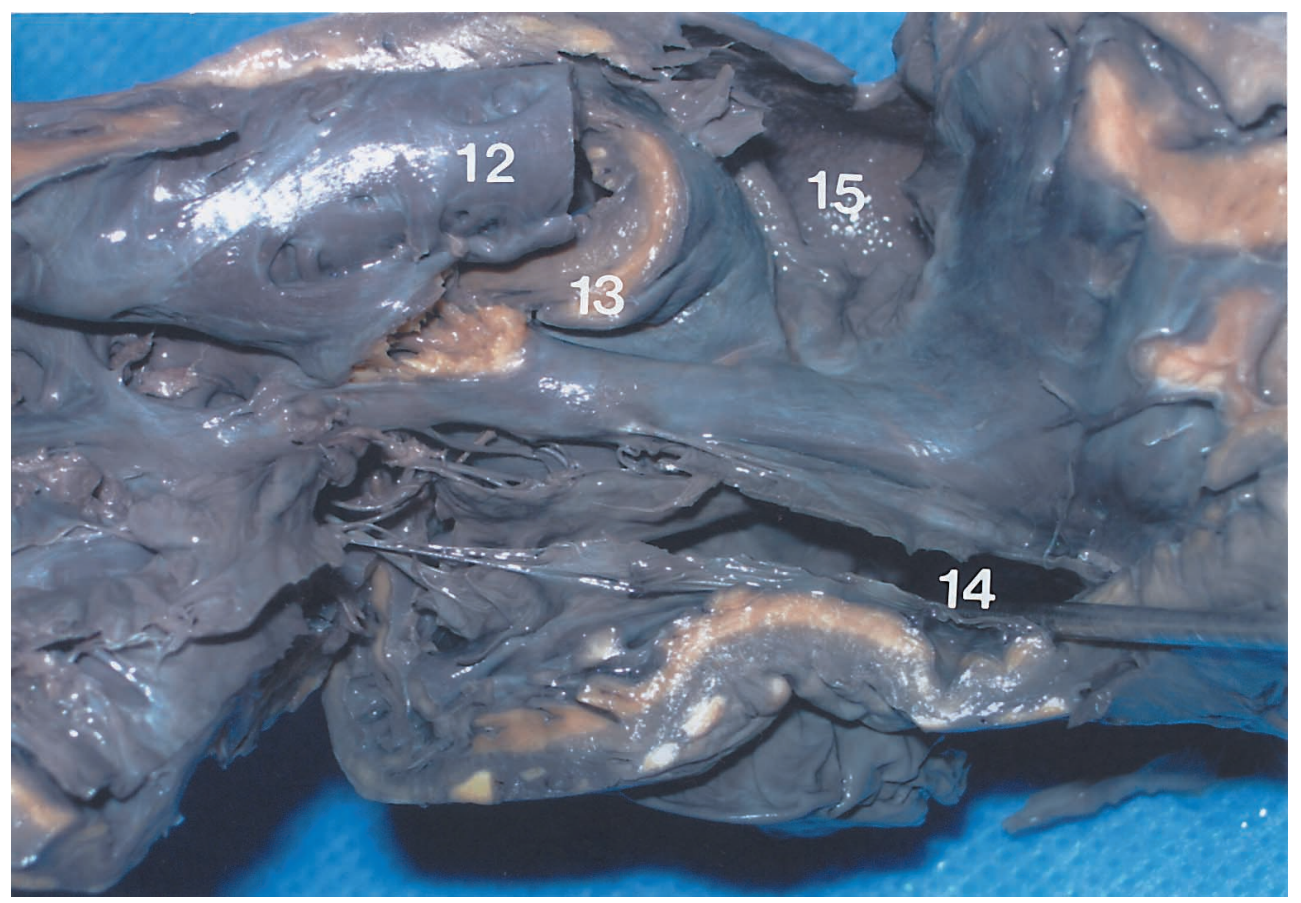

Ambos surcos interventriculares se continuaban entre sí en el ápex del corazón.

Los bordes ventriculares derecho e izquierdo se situaban, respectivamente, a la derecha y a la izquierda del animal. Ambos constituían los bordes derecho e izquierdo del contorno cardíaco y eran ligeramente oblicuos de lateral a medial llegando al ápex. La longitud promedio de estos bordes era de $6,0 \mathrm{~cm}$, desde el surco coronario hasta el ápex.

Fig. 5. Vista interior del ventrículo derecho incidido del corazón de Pontoporia blainvillei.

tía ventralmente al tronco pulmonar, y en su centro a la aorta que aparecía a la izquierda del tronco pulmonar. Ambos vasos estaban unidos por el ligamento arterioso. Dorsalmente y a la derecha llegaban las venas cavas al atrio derecho y tres venas pulmonares unidas en un tronco común al atrio izquierdo (3, Fig. 3).
El ápex del corazón de Pontoporia blainvillei correspondía a ambos ventrículos.

En la conformación interior del atrio derecho destacaba el gran desarrollo de la cresta terminal y la fosa oval (10 y 11, Fig. 4). La fosa oval se observaba como una depre- 
sión infundibuliforme de $10,0 \mathrm{~mm}$ de diámetro en su entrada. La aurícula derecha presentaba los músculos pectinados (9, Fig. 4) irradiados desde la cresta terminal. Tres aberturas venosas, correspondientes a ambas venas cavas y al seno coronario, se abrían en el atrio derecho; caudalmente se ubicaba el orificio atrioventricular derecho. La valva atrioventricular derecha tenía tres cúspides.

En el interior del ventrículo derecho (Fig. 5) los músculos papilares (12, Fig. 5) se observaron distribuidos en subarteriosus, magnus y parvi. En la parte vecina del ápex había gran número de trabéculas carnosas, dándole un aspecto enrejado o de encaje, al ventrículo. Una trabécula septomarginal que medía $11,0 \mathrm{~mm}$ de largo, se extendía entre el músculo papilar subarteriosus y el músculo papilar magnus. Ventral a ella existe una trabécula delgada en forma de Y entre el M. papilar magnus y el menor. La cresta supraventricular era bastante gruesa y separaba los orificios atrioventriculares derecho y pulmonar. La valva pulmonar tenía tres válvulas. El septum interventricular en su parte central tenía $12 \mathrm{~mm}$ de espesor.

El atrio izquierdo no tenía nada de particular, excepto que llegaban a él, las tres venas pulmonares unidas en un tronco común (látex rojo, 3, Fig. 3). La valva atrioventricular izquierda estaba formada por dos cúspides y la valva aórtica por tres. En el ventrículo izquierdo habían dos músculos papilares bien desarrollados.

En los corazones estudiados se observó al realizar cortes la presencia de grasa subendocárdica (13, Fig. 5). El corazón estaba irrigado por las arterias coronarias derecha e izquierda que surgían de los respectivos senos de la aorta. La arteria coronaria izquierda era de mayor calibre y se distribuía a la mayoría del territorio cardíaco.

La arteria coronaria izquierda tenía un trayecto de $0,9 \mathrm{~mm}$ y se dividía en arterias paraconal y circunfleja.

La rama interventricular paraconal (7, Fig. 2) contra el origen del tronco pulmonar, describía en todos los ejemplares examinados, una curva de convexidad izquierda, sobre un trayecto mayor a 1,0 cm, después la arteria se tornaba más sinuosa, pero rectilínea, a lo largo del surco interventricular paraconal, y emitía ramas hacia ambos ventrículos, mayormente hacia el ventrículo izquierdo. En la mitad de su trayecto, emitía la rama principal hacia el ventrículo derecho. En cambio, hacia el ventrículo izquierdo las dos ramas principales salen en los tercios proximal y distal de la arteria paraconal.

En el ápex se anastomosaban las ramas interventriculares paraconal y subsinusal, describiendo una leve curva de convexidad izquierda, de unos 5,0 mm de longitud visible desde la cara auricular.

A través del surco coronario y hacia la izquierda transitaba la rama circunfleja con un trayecto algo flexuoso que, al llegar al surco interventricular subsinusal, daba la rama subsinusal (8, Figura 3). La rama circunfleja iba emitiendo ramas hacia ambas caras del ventrículo izquierda mayormente sobre la cara arterial del corazón y emitía una rama del borde ventricular derecho.

La arteria coronaria derecha tenía menos de la mitad de tamaño que la izquierda y se colocaba entre la aurícula derecha y el tronco pulmonar, emitiendo una rama hacia el cono arterioso, que se dividía una contra la base del tronco pulmonar y otra que se dirigía hacia distal en el ventrículo derecho. Posteriormente, la arteria continuaba por el surco coronario cubierta por la aurícula derecha y emitía una rama por el tercio proximal del borde ventricular izquierdo.

En todos los corazones estudiados las ramas interventriculares paraconal y subsinusal se anastomosaban en el ápex y habían anastomosis de delgado calibre entre ramas de ambas coronarias.

En dos corazones estudiados fue observada una variante de los resultados anteriores de irrigación. La arteria paraconal emitía una rama que se ubicaba dorsalmente a ella y que, en las proximidades del ápex, volvía a unírsele. Algo similar ocurría con la arteria circunfleja, que surgía como dos vasos, uno principal y otro más pequeño que se le unía tras un trayecto corto, ambos vasos cubiertos por la aurícula izquierda.

Las venas cardíacas transitaban junto a las arterias y conducían la sangre hacia el seno coronario. Éste se abría en el atrio derecho próximo al orificio de la vena cava caudal y recibía a la vena cordis magna y a la vena cordis media. La vena cordis magna transitaba primero por el surco interventricular paraconal y luego por el surco coronario, recibiendo afluentes ventriculares y atriales, respectivamente. La vena cordis media recorría el surco interventricular subsinusal a la izquierda de la arteria.

\section{DISCUSIÓN}

La disposición de las tres partes de la cavidad torácica, una cardíaca ventral y dos pulmonares separadas dorsalmente por el mediastino dorsal, recuerda la disposición embrionaria. Los pulmones no crecen hacia ventral rodeando al corazón, sino que su crecimiento parece ser 
mayormente en sentido cráneo-caudal, motivo por el cual ambas cavidades pleurales mantienen su posición dorsal y el corazón y pericardio quedan solos situados ventralmente. Estudiar el desarrollo y evolución de la cavidad torácica, sus órganos y la disposición de sus serosas, implica obtener animales en varias etapas de desarrollo fetal.

Los corazones de Pontoporia blainvillei que hemos descrito tipifican al corazón de mamífero en aspectos generales. Sin embargo, la disposición y la topografía en la cavidad torácica es muy diferente. La cara auricular es ventral, la que en los mamíferos domésticos está dirigida hacia el lado izquierdo de la pared torácica. La cara atrial es dorsal y en los mamíferos domésticos es derecha. El mismo criterio adoptado por la NAV debería aplicarse en éste y otros Cetáceos, cara atrial, por corresponder a la cara donde se observa la mayor parte de los atrios y cara auricular por ser donde se observan mayormente ambas aurículas. Igual a lo que indican Ochrymowych et al. (1984) para el corazón de la ballena minke y como vimos nosotros en un corazón colectado de otra ballena de la misma especie. En la mayoría de los corazones del Pontoporia blainvillei la diferencia anatómica más notoria es el aplastamiento dorsoventral del corazón con sus ventrículos colocados lado a lado. Tarpley et al. (1997) estudiaron ejemplares de Balaena mysticetus y son concordantes con la disposición de las caras en Pontoporia blainvillei, sin embargo estos autores utilizaron, además, los nombres de caras diafragmática y esternocostal para las caras atrial y auricular. Debido a criterios topográficos y por ser confusos estos nombres, no son adecuados para Pontoporia blainvillei. Solamente la cara auricular se podría considerar esternocostal, la cara atrial no se relaciona al diafragma.

En otra investigación que realizamos fue la misma forma obervada en un corazón de delfín Fraser (Lagenodelphis hosei), si bien el corazón de este delfín no era tan aplastado dorsoventralmente.

Ochrymowych et al., describieron un corazón de Balaenoptera acutorostrata. Estos autores citan varios trabajos previos de corazones de ballenas, correspondiendo a observaciones muy antiguas.

La disposición del pericardio coincide con la descripción hecha en el corazón de Phocaena phocaena por Rowlatt et al. (1975), aunque estos autores no mencionan nada similar a los fondos de saco que forma, la cavidad pericárdica cranealmente a las aurículas, como observamos en Pontoporia blainvillei.

En relación al conducto arterioso, reportamos sólo la existencia del ligamento arterioso, al igual que Johansen et al. (1988), quienes mencionaron que ha sido indicado que permanece abierto en cetáceos adultos normales. Examinando los corazones de dos ballenas piloto estos autores no hallaron persistencia del ductus arteriosus, encontrando un lumen totalmente obliterado.

En la conformación interior del órgano y en particular del ventrículo derecho, lo más llamativo es la gran cantidad de trabéculas carnosas y la disposición de las trabéculas septomarginales, la mayor de las cuales no merecería este nombre pues se extendían entre los músculos papilares.

Para el caso de las valvas cardíacas en general, nos guiamos también por la NAV, usando los nombres de Valvas atrioventricularis dextra y sinistra con Cuspis y Valva Trunci Pulmonalis y Valva Aortae con sus válvulas semilunares. Los nombres dados por la NAV a las cúspides y valvas están acorde a su disposición en Pontoporia blanvillei.

En relación a la circulación coronaria en Pontoporia blainvillei, predominaba en tamaño y territorio de distribución la arteria coronaria izquierda. En la mayoría de las ballenas ambas arterias coronarias son de igual tamaño y distribución (Truex et al., 1961).

En las ballenas hasn sido observadas abundantes anastomosis intercoronarias (Truex et al.). En Pontoporia blainvillei era constante las anastomosis de las ramas interventriculares paraconal y subsinusal en el ápex y de vasos menores; en dos especímenes existía una división de los vasos paraconal y circunflejo. Las anastomosis arteriovenosas descritas en ballenas (Truex et al.) no fueron vistas en Pontoporia blainvillei.

La disposición de la circulación venosa es similar a la descrita Truex et al. y similar, aunque más simple que en los mamíferos domésticos

De la anatomía cardíaca macroscópica de este animal falta estudiar su irrigación linfática, su sistema de conducción e inervación. El sistema de conducción cardíaco en Lagenorhynchus albirostris ha sido disecado y descrito en detalle (Van Nie).

AGRADECIMIENTOS: Los autores agradecen al Grupo Cetáceos Uruguay por proveernos los materiales y a los Dres. H. Katz y R. Möller por incentivarnos a estudiar los animales marinos. También agradecemos al Prof. Eduardo Martin de la Universidad Nacional de Río Cuarto, por su asistencia en nuestras consultas. 
PÉREZ, W. \& LIMA, M. Cardiac anatomy of the Pontoporia blainvillei. Int. J. Morphol., 24(3):351-356, 2006.

SUMMARY: The aim of this study is to describe the external and internal conformation and the arterial irrigation of La Plata Dolphin's heart (Pontoporia blainvillei). Twelve animals obtained from nets of fishing ships were used and their hearts were studied by means of simple dissection. The pericardium was fixed caudally to the diaphragm, ventrally to the thoracic transverse muscle and laterally to the ribs and intercostal muscles. The heart weighted about 115 grams, was flattened dorsoventrally, its base was cranial, its apex was shared by both ventricles. The ventral face corresponds to the auricular face of the Nomina Anatomica Veterinaria (NAV), and the dorsal face corresponds to the atrial face. In the internal conformation of the right atrium the great development of the crista terminalis and the fossa ovalis stood out. Inside the right ventricle the papillaris muscles were observed distributed in subarteriosus, magnus and parvi. The dextra septomarginal trabecules were multiple and situated between the papillaris muscles. In the left ventricle they had two well developed papillaris muscles. The left coronary artery is the most important vessel in the arterial irrigation of the cardiac territory.

KEY WORDS: La Plata dolphin; Heart anatomy; Cardiology; Coronary arteries.

\section{REFERENCIAS BIBLIOGRÁFICAS}

Johansen, K.; Elling, F. \& Paulev, P. E. Ductus arteriosus in pilot whales. Jpn. J. Physiol., 38(3):387-92, 1988.

Kamiya, T. \& Yamasaki, F. Organ weights of Pontoporia blainvillei and Platanista gangetica (Platanistidae). Sci. Rep. Whales Res. Inst., 26:265- 70, 1974.

Nomina Anatomica Veterinaria. Fifth edition. Prepared by the International Committee on Veterinary Gross Anatomical Nomenclature (I.C.V.G.A.N.) and authorized by the General Assembly of the World Association of Veterinary Anatomists (W.A.V.A.). Knoxville, TN (U.S.A.) 2003. Published by the Editorial Committee. Hannover, Columbia, Gent, Sapporo. 2005. http:// www.wava-amav.org/Downloads/nav_2005.pdf

Ochrymowych, C. \& Lambertsen, R. Anatomy and vasculature of a minke whale heart. Am. J. Anatomy, $169(2): 165-75,1984$.

Rowlatt, U. \& Gaskin, D. E. Functional anatomy of the heart of the harbor porpoise, Phocaena phocaena. J. Morph. 146:479-94, 1975.

Tarpley, R. J.; Hillmann, D. J.; Henk, W. G. \& George J. C. Observations on the external morphology and vasculature of a fetal heart of the bowhead whale, Balaena mysticetus. Anat. Rec., 247(4):556-81, 1997.

Truex, R. C. et al. Anatomy and pathology of the whale heart with special reference to the coronary circulation. Anat. Rec., 141:325-53, 1961.

Van Nie, C. J. The conducting system of the heart of the White-Snouted dolphin Lagenorhynchus albirostris (Gray, 1846). Lutra, 28:106-12, 1985.

\author{
Correspondence to: \\ Dr. William Perez \\ Área de Anatomía \\ Facultad de Veterinaria \\ A. Lasplaces 1550 \\ 11600. Montevideo \\ URUGUAY \\ Tel: ++59826234163 \\ Fax: ++598 26222933 \\ Email: vetanat@gmail.com
}

Recibido : 17-03-2006

Aceptado: 16-06-2006 